\title{
Effect of Shikonin on Spinal Cord Injury in Rats Via Regulation of HMGB1/TLR4/NF- $\kappa$ B Signaling Pathway
}

\author{
Yihui $\mathrm{Bi}^{\mathrm{a}}$ Yapeng Zhu ${ }^{\mathrm{a}}$ Mingkai Zhang ${ }^{\mathrm{a}, \mathrm{d}} \quad$ Keke Zhang $^{\mathrm{a}} \quad$ Xingyi Hua ${ }^{\mathrm{a}}$ \\ Zheng Fang ${ }^{a}$ Jian Zhou ${ }^{a}$ Wenjie Daia Yixing Cuia Jun Lic Tao You ${ }^{a}{ }^{a, b, c}$ \\ aDepartment of orthopaedic, The First Affliated hospital, Anhui Medical University, HeFei, ${ }^{b}$ Anhui \\ Provincial Hospital, Anhui Medical University, HeFei, 'College of Pharmacy, Anhui Medical University, \\ HeFei, dDepartment of orthopaedic, Tongling pepole's hospital, TongLing, China
}

\section{Key Words}

Shikonin • SCI • Inflammation response • Apoptosis • HMGB1/TLR4/NF-KB signaling pathway

\begin{abstract}
Background/Aims: Shikonin, a compound extracted from Zicao, has been demonstrated to hold anti-bacterial, anti-inflammatory, and anti-tumor activities in various diseases and it has been shown to protect human organs from injuries. However, the effect of shikonin on the recovery of spinal cord injury (SCI) remains unknown. This study was designed to estimate the potential therapeutic effect and underlying mechanism of shikonin on SCI in vivo. Methods: In the study, we used HE staining, ELISA assay, transfection assay, TUNEL assay, real time PCR and Western blot to detect the effects of shikonin on spinal cord injury in rats. Results: we showed that shikonin could promote the recovery of motor function and tissue repair after SCI treatment in rats SCI model. Moreover, we demonstrated that shikonin inhibited the spinal cord edema in SCI model of rats. According to further investigation, shikonin induced the reduction of inflammatory response through decreasing the expression levels of HMGB1, TLR4 and NF-KB after SCI injury. In addition, we also found that shikonin could suppress the apoptosis and expression of caspase-3 protein in SCI model of rats. Conclusion: Our results demonstrated that shikonin induced the recovery of tissue repair and motor function via inactivation of HMGB1/TLR4/NF-KB signaling pathway in SCI model of rats. Meanwhile, shikonin regulated the inflammation response in SCI by suppressing the HMGB1/TLR4/NFKB signaling pathway. The described mechanism sheds novel light on molecular signaling pathway in spinal cord injury and secondary injury including inflammatory response.
\end{abstract}

Jun Li,

and Tao You

KARGER
Department of orthopaedic, The First Affliated hospital, and College of Pharmacy, Anhui Medical University, 218 Jixi Road, HeFei, (China)

E-Mail youtao@21cn.com, lijun@ahmu.edu.cn 


\section{Cellular Physiology Cell Physiol Biochem 2017;43:481-491

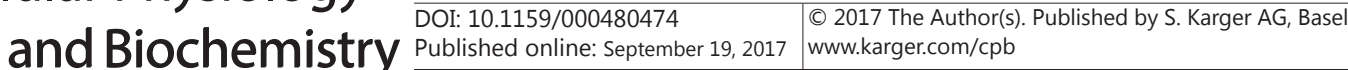 \\ Bi et al.: Molecular Mechanism of Shikonin in Spinal Cord Injury}

\section{Introduction}

Spinal cord injury (SCI) is the traumatic damage to the cervical spinal cord involving hypoxia, lipid peroxidation and apoptosis $[1,2]$. SCI injuries consist of the primary injury and the secondary injury, which primarily originate from the injury of traction and cerebral trauma respectively [3]. SCI results in an infaust outcome to the physical and psychological injury involving metabolic disturbance of the extracellular matrix (ECM) and inflammatory response [4,5]. Excessive inflammation response set barrier to the nerve repair and regeneration [6]. Although current treatments of SCI including drugs, surgery and hyperbaric oxygen intervention partial effectively alleviate SCI injury, side effects and secondary injury still disturb the SCI patient $[7,8]$. Therefore, a new route and effective manner of cure SCI injury especially secondary injury need to be further explored.

Shikonin, extracted from the plant root of Lithospermum erythrorhizon, is a traditional Chinese herbal medicine in China for thousands of years [9]. Shikonin was well illustrated for its anti-viability effect in various cancer cells [10]. Previous study found shikonin could remarkably inhibit the carrageenan-induced paw edema and the development in chronic arthritis [11]. NF- $\kappa B$ is recognized as a key mediator in many aspects of SCI, including the innate immune response and inflammation [12]. NF- $\mathrm{KB}$ is necessary for the expression of tumor necrosis factor- $\alpha$ (TNF- $\alpha$ ), interleukin-1 $\beta$ (IL-1 $\beta$ ), and interleukin-6 (IL-6) inflammatory cytokines [13]. Previous report demonstrated that NF- $\kappa B$ signaling pathway could be suppressed by shikonin, which associated with reduction of oedema induced by phorbol ester through interfering with IкB $\alpha$ degradation in macrophages [14]. Further study revealed shikonin inhibited NF- $\mathrm{KB}$ activation via up-regulation of PI3K/Akt/Nrf2 signaling pathway in EA.hy926 endothelial cells [15]. However, the molecular mechanism of shikonin interaction with NF- $\kappa \mathrm{B}$ pathway in the alleviation process of SCI remains unknown.

High mobility group protein B1 (HMGB1), a mediator of sepsis, is a new intervention factor to suppress inflammation [16]. Previous study revealed that HMGB1 could activate inflammation response through interaction with TLR receptor and NF- $\kappa B$ in the secondary SCI injury [17]. In the other study, shikonin inhibited the expression of HMGB1 through IFN and NF-KB signaling pathways in RAW264.7 cells [18]. Although HMGB1 has been testified to be relevant with the initiation and progress of the secondary SCI, the molecular mechanisms of HMGBI on the alleviation process of SCI and the control of excess inflammatory cytokines, and the relationship with shikonin were still obscure.

Various protein expression levels and signaling pathways have been involved in the antiinflammation role of shikonin in various human diseases, but the detailed molecular and physiological mechanisms underlying the process remain obscure. Whether shikonin has an anti-inflammation effect on the progress SCI, and the relevant molecular mechanism remains to be solved. Here, we demonstrated that shikonin improved the recovery of locomotor function and tissue repair via inhibiting the expression of HMGB1 and apoptosis of the spinal cord tissue in vivo. In addition, we further showed shikonin suppressed inflammation

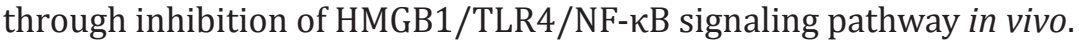

\section{Materials and Methods}

Drugs and chemicals

Shinkonin was purchased from Nanjing Tcm Institute of Chinese Material Medica (Nanjing, China). Methylprednisolone was provided by Pfizer (NV, Belgium) TNF- $\alpha$, IL-1 $\beta$ and IL-6 ELISA kits were supplied by R\&D Systems (BiosPacific, CA, USA). HMGB1, TLR4, NF- $\kappa$ B antibodies were purchased from Cell Signaling (Massachusetts, USA).

Animals

The protocols of animal use and care conformed to Guide for the Care and Use of Laboratory Animals from the National Institutes of Health, and all experimental protocols described in this study were approved 


\section{Cellular Physiology Cell Physiol Biochem 2017;43:481-491 \begin{tabular}{l|l|l} 
and Biochemistry 10.1159/000480474 & $\begin{array}{l}\text { (c) } 2017 \text { The Author(s). Published by S. Karger AG, Basel } \\
\text { www.karger.com/cpb }\end{array}$
\end{tabular}}

Bi et al.: Molecular Mechanism of Shikonin in Spinal Cord Injury

by the Ethics Review Committee for Animal Experimentation of Anhui Medical University. Male SpragueDawley (SD) rats weighing 180 to $220 \mathrm{~g}$ were acquired from Animal Center of Chinese Academy of Sciences, Shanghai, China. Animals were housed at the animal facility of the Laboratory Animals Centre of Anhui Medical University.

\section{Animal model of SCI and experimental groups}

Anesthesia was induced with $4 \%$ isoflurane and maintained with $2 \%$ isoflurane in $98 \% \mathrm{O}^{2}$. Throughout the procedure, the depth of sedation was monitored by an absent response to a toe pinch. A laminectomy was performed at the thoracic vertebra level 10 (T10) after shaving and cleaning in an incubator $\left(37.5^{\circ} \mathrm{C}\right)$ until fully recovered from the anesthesia and then each rat was housed individually. A moderate contusion injury was induced using a modified Allen's weight drop apparatus ( $8 \mathrm{~g}$ weight at a vertical height of $40 \mathrm{~mm}$, $8 \mathrm{~g} \times 40 \mathrm{~mm}$ ) on the spinal cord, as previously described [19]. Sham-operated animals were only subjected to laminectomy. After surgery, the muscles were sutured in layers and the skin incision was closed with 3-0 silk threads. Penicillin G (40, 000 U, i. m.) was administrated daily for 3 days to prevent infection. The experimental groups consisted of five groups $(\mathrm{N}=8)$ : sham group, exposed the same area without injury; SCI group, performed with spinal cord injury; Methylprednisolone (MPSS) group, performed with spinal cord injury and treated with $100 \mathrm{mg} / \mathrm{kg}$ of MPSS, administered by intraperitoneal injection 30 minutes after injury; Shi (10mg) group, performed with spinal cord injury and treated with $10 \mathrm{mg} / \mathrm{kg}$ of shikonin, , administered by intraperitoneal injection 30 minutes after injury; Shi (100mg) group, performed with spinal cord injury and treated with $100 \mathrm{mg} / \mathrm{kg}$ of shikonin, , administered by intraperitoneal injection 30 minutes after injury. To investigate the mechanism of shikonin regulated in rats after SCI injury. Rats were randomly divided into six group ( $\mathrm{N}=8$ ): sham group, exposed the same area without injury; SCI group, performed with spinal cord injury; MPSS group, performed with spinal cord injury and treated with 100 $\mathrm{mg} / \mathrm{kg}$ of MPSS, administered by intraperitoneal injection 30 minutes after injury; MPSS + HMGB1 group, performed with spinal cord injury and treated with $100 \mathrm{mg} / \mathrm{kg}$ of MPSS and Ad-HMGB1, administered by intraperitoneal injection and subcutaneous respectively 30 minutes after injury; Shi group, performed with spinal cord injury and treated with $100 \mathrm{mg} / \mathrm{kg}$ of shikonin, administered by intraperitoneal injection 30 minutes after injury; Shi + HMGB1 group, performed with spinal cord injury and treated with $100 \mathrm{mg} / \mathrm{kg}$ of shikonin and Ad-HMGB1, administered by intraperitoneal injection and subcutaneous respectively 30 minutes after injury.

\section{Behavioral assessment}

Three rats from each group were subjected to locomotor activity evaluation at $24 \mathrm{~h}, 48 \mathrm{~h}$ and $72 \mathrm{~h}$ post-injury using the Basso, Beattie, and Bresnahan (BBB) score method. Two independent and well-trained testers observed movement of each rat for $4 \mathrm{~min}$ and scored motor functions according to BBB scales [20]. The final score for each animal was obtained by averaging values from both investigators. Rats with perineal infections, limb wounds, or tail and foot grazing were eliminated from the test.

\section{Water Content}

Spinal cord edema was assessed by water content of the spinal cord tissue. Briefly, $72 \mathrm{~h}$ post injury, the spinal cords were dried for $48 \mathrm{~h}$ at $-80{ }^{\circ} \mathrm{C}$, which used to detect dry weight. Water content in spinal cords was calculated by the following formula: water content of spinal cord $(\%)=($ wet weight - dry weight $) /$ wet weight $\times 100 \%$.

\section{HE staining}

The $5 \mu \mathrm{m}$ animal sections were made from the paraffin embedded blocks of the formalin-fixed spinal cord specimens, which were harvested from the animals from each group were subjected to routine HE staining and evaluated microscopically. Images were collected at 400X magnification.

\section{ELISA analysis}

Rats were sacrificed by transcardiac perfusion with cold PBS to eliminate RNA and protein expressed by blood cells. The spinal cord was immediately dissected on ice. Thereafter, spinal cord segments containing the injury epicenter were removed as quickly as possible. Rats from each group were subjected to mouse IgG measurements from $72 \mathrm{~h}$ post-injury by ELISA. All assays were performed in duplicates using recommended buffers, diluents, and substrates. 


\section{Cellular Physiology Cell Physiol Biochem 2017;43:481-491 \begin{tabular}{ll|l} 
and Biochemistry 10.1159/000480474 & $\begin{array}{l}\text { (C) } 2017 \text { The Author(s). Published by S. Karger AG, Basel } \\
\text { www.karger.com/cpb }\end{array}$ \\
\hline
\end{tabular}}

Bi et al.: Molecular Mechanism of Shikonin in Spinal Cord Injury

RNA extraction and quantitative real-time polymerase chain reaction ( $q P C R$ ) analysis

Rats were sacrificed, flash-frozen and stored in liquid nitrogen for subsequent RNA and protein extraction. Total RNA was extracted using the TRIzol @ reagent (Invitrogen, Carlsbad, USA). HMGB1: forward primer (5'-CAAACCTGCCGGGAG GAGCA-3'); reverse primer (5'-TCTTTCATAACGAGCCTTGTCAGCC-3'), TLR4: forward primer (5'-TATCCAGAGCCGTTGGTGTA-3'); reverse primer (5'-CCCAC TCGAGGTAGGTGTTT-3'), NFкB: forward primer (5'-CCTAGCTTTCTCTGAAC TGCAAA-3'); reverse primer (5'-GGGTCAGAGGCCAATAGAGA3'),GAPDH: forward primer (5'-GGTGAAGGTCGGTGT GAACG-3'); reverse primer (5'-CTCG CTCCTGGAAGATGGTG-3'), qPCR analyses were performed with LightCycler 480 SYBR Green I Master (Roche, Welwyn Garden, Swiss).

\section{Western blot}

Proteins were separated on a $10 \%$ sodium dodecyl sulfate-polyacrylamide gel and transferred on to a nitrocellulose membrane (Bio-Rad, Hercules, USA). Then, the membrane was blocked with 5\% non-fat milk and incubated with primary antibodies. The proteins were detected using enhanced chemiluminescence reagents (Thermo Scientific).

\section{Adenovirus vector construction, infection and transfection}

Clone vector of HMGB1 was provided from Sangon Biotech (Shanghai). HEK-293T cells were plated in culture dish. After HEK-293T cells attached to the plate overnight, the linearized DNA, the shuttle vector and pacAd vector were cotransfected into HEK-293T by Lipofectamine 2000 (Invitrogen). Viral lysates were harvested, purified and tittered. One day prior to infection, cells were seeded in plate. Next day adenoviruses were added into the medium and infecting the cells.

\section{TUNEL assay}

Sections were deparaffinized, antigen retrieval was performed with proteinase $\mathrm{K}$ at $37^{\circ} \mathrm{C}$ for 30 minutes, then incubation with $20 \%$ fetal calf serum and $3 \%$ bovine serum albumin at $37^{\circ} \mathrm{C}$ for 15 minutes. The reaction was terminated with $5 \mu \mathrm{L}$ TdT and $45 \mu \mathrm{L}$ fluorescein-labeled oligodeoxynucleotide buffer for 30 min at $37^{\circ} \mathrm{C}$. Endogenous peroxidase was blocked with $0.3 \% \mathrm{H}_{2} \mathrm{O}_{2}$, immunoreaction with POD at $37^{\circ} \mathrm{C}$ for 30 minutes, the sections were incubated with $20 \%$ fetal calf serum, $3 \%$ bovine albumin serum and $1 \%$ blocking agent at $37^{\circ} \mathrm{C}$ for 15 minutes. Staining was visualized with $\mathrm{DAB}-\mathrm{H}_{2} \mathrm{O}_{2}$. The sections were counterstained with hematoxylin, dehydrated through an ethanol series, rendered transparent using xylene, and mounted. Apoptotic cells were counted under an optical microscope (Olympus, Tokyo, Japan) at 200× magnification.

\section{Immunohistochemistry}

Sections were incubated in a hydrogen peroxide solution for 1 hour at room temperature. IBA-1 (1:400, Abcam) and GFAP (1:500, Sigma) antibodies were used to evaluate microglial activation and reactive gliosis in the injured spinal cord. The sections were visualized using confocal microscopy (Zeiss 710 and LSM software).

\section{Statistical analysis}

Results are presented as the means \pm S.D. from at least 3 independent experiments. ${ }^{*} \mathrm{P}<0.05$ versus SCI group, ${ }^{* *} \mathrm{P}<0.01$ versus SCI group, ${ }^{* * *} \mathrm{P}<0.001$ versus $\mathrm{SCI}$ group. The statistical differences were calculated by the Student's $t$-test or one-way ANOVA analysis of variance with Dunnett's test.

\section{Results}

\section{Shikonin Improves the recovery of Locomotor function and tissue repair}

Basso Beattle Bresnahan (BBB) scores were evaluated for the recovery of locomotor function after SCI injury in different groups. We found the BBB score in SCI group was remarkably lower than other groups at different time points. The BBB score in the shikonin treatment group at different doses was significantly higher than SCI treatment rats at selected time points (Fig. 1). The recovery level of locomotor function after SCI injury between MPSS and $100 \mathrm{mg} / \mathrm{kg}$ of shikonin group had no significant difference. We also tested the tissue 


\section{Cellular Physiology Cell Physiol Biochem 2017;43:481-491

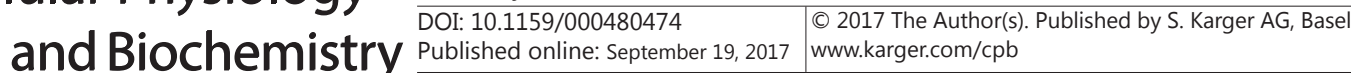

Fig. 1. Basso, Beattie and Bresnahan (BBB) scores of rats in each group at different time points after injury. The BBB scores were evaluated at 24, 48, 72 hours after sham surgery or spinal cord injury, $n=8$ /group for all the groups. Results are expressed as the mean \pm S.D. from three independent experiments. ${ }^{* *}$ $\mathrm{P}<0.01$ versus SCI group, $* * * \mathrm{P}<0.001$ versus SCI group.

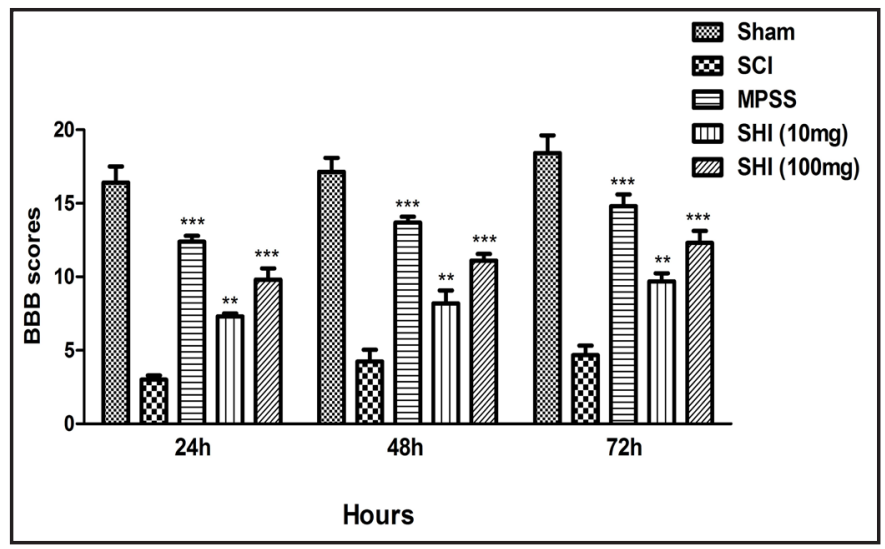

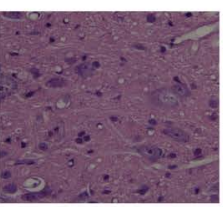

Sham

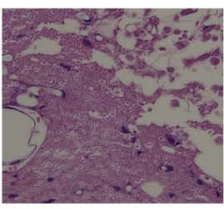

$\mathrm{SCl}$

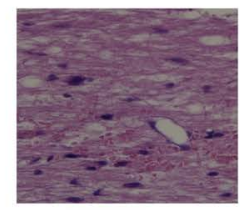

MPSS

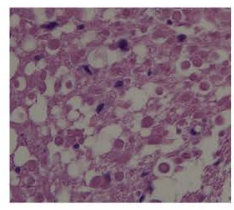

SHI(10mg)

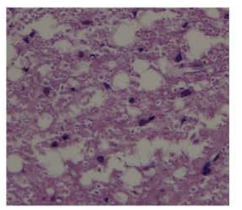

SHIl100mg)

Fig. 2. Effects of shikonin on the tissue repair in the spinal cord in rats with spinal cord injury. The spinal cord tissue harvested at $72 \mathrm{~h}$ after injury was subject to HE staining. The results presented are representative.

repair after at $72 \mathrm{~h}$ after SCI injury by HE staining. The data were collected to testify the effect of shiknin on the spinal cord tissue recovery. The histological tissue injuries including loose structure and neutrophil infiltration were obviously relieved by MPSS and shikonin with different doses treatment (Fig. 2). In addition, SCI injury enhanced IBA-1 and GFAP immunoreactivity compared with control group, while IBA-1 and GFAP intensity obviously attenuated by MPSS and shikonin with different doses treatment (Fig. 7a and b).

Shikonin diminishes the water content and inhibits the inflammation levels after SCI injury

We next investigated the water content of spinal cord in different groups (Fig. 3a). The results showed that the water content of SCI group was obvious increased compared with sham group. However, after treatment with different doses of shikonin, the mean value of water content was significantly reversed, which suggested that shikonin treatment could alleviate SCI-induced spinal corded ema in rats. Furthermore, we also detected the inflammatory cytokines after SCI injury at different groups. The expression of IL-1 $\beta$, IL-6 and TNF- $\alpha$ at $72 \mathrm{~h}$ post-injury were evaluated using ELISA. As expected, the cytokine levels of SCI group were obviously increased compared with sham group. In addition, different doses of shikonin treatment could dramatically decrease IL-1 $\beta$ (Fig. 3b), IL-6 (Fig. 3c) and TNF- $\alpha$ (Fig. 3d) expression compared with SCI group. The results revealed that shikonin could inhibit inflammation levels after SCI injury.

Shikonin reduced the mRNA and protein expressions of HMGB1, TLR4 and NF- $\kappa B$ after SCI injury

To investigate the effect of shikonin on the HMGB1/TLR4/NF- $\kappa B$ signaling pathway, the mRNA and protein levels of HMGB1, TLR4 and NF- $\kappa B$ were evaluated after SCI injury. We found the mRNA level of HMGB1 in shikonin treatments were significantly lower than SCI group, especially in the $100 \mathrm{mg} / \mathrm{kg}$ of shikonin group (Fig. 4b). Furthermore, the TLR4 mRNA level in the $100 \mathrm{mg} / \mathrm{kg}$ of shikonin group was obvious decreased compared with 
Fig. 3. SHI diminishes the water content and inhibits the inflammation levels after SCI. (a) SHI treatment significantly attenuated SCI-induced spinal corded ema in rats. Quantification data of IL-1 $\beta(b)$, IL-6 (c), TNF- $\alpha(d)$ production in the spinal cord at $72 \mathrm{~h}$ after injury, as assessed by ELISA. Results are expressed as the mean \pm S.D. from three independent experiments.

a

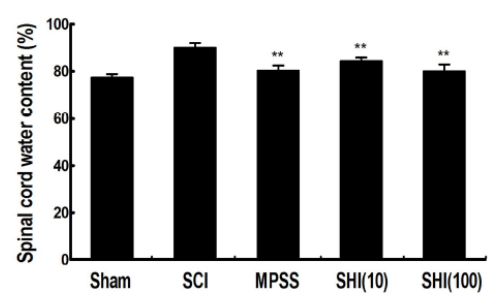

C

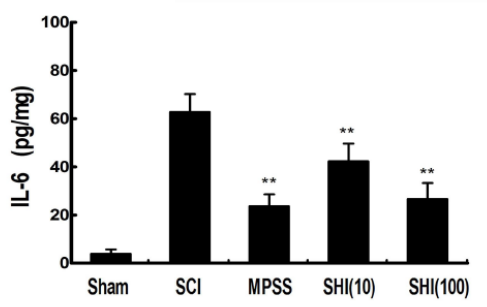

b

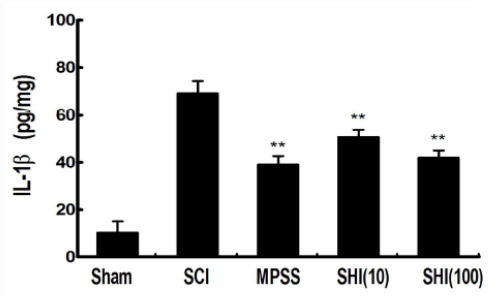

d

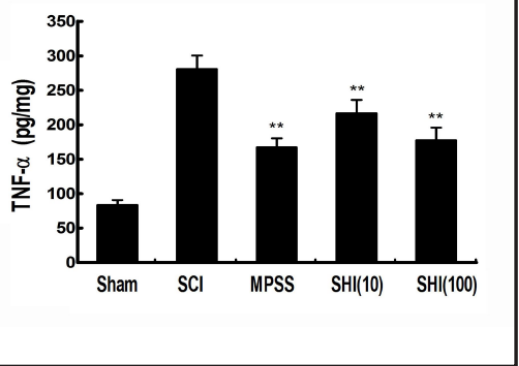
$\mathrm{P}<0.01$ versus SCI group.

Fig. 4. Expression of HMGB1, TLR4, $\mathrm{NF}-\kappa \mathrm{B}$ after SCI injury in each group. (a) The protein levels of HMGB1, TLR4, NF-кB at 72 $h$ post injury. The mRNA expression of HMGB1(a), TLR4 (b), NF- $\mathrm{B}$ (c) and at $72 \mathrm{~h}$ post injury. Results are expressed as the mean \pm S.D. from three independent experiments. * $\mathrm{P}<0.05$ versus SCI group, ** $\mathrm{P}<0.01$ versus SCI group.
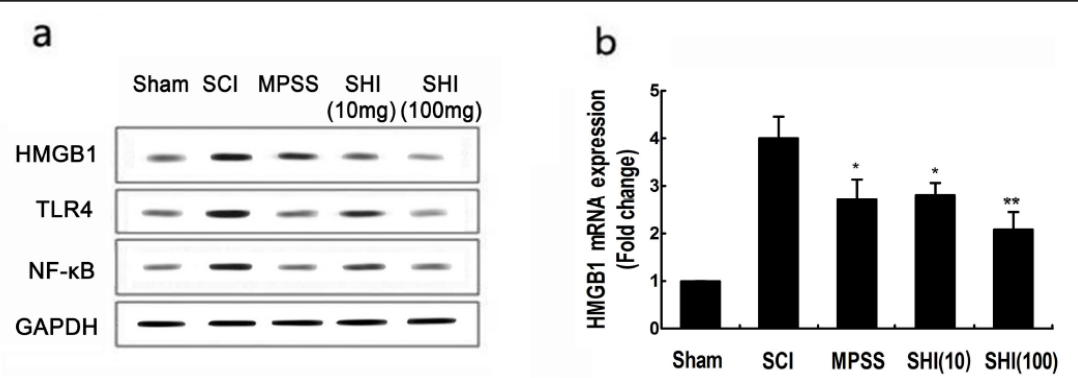

C

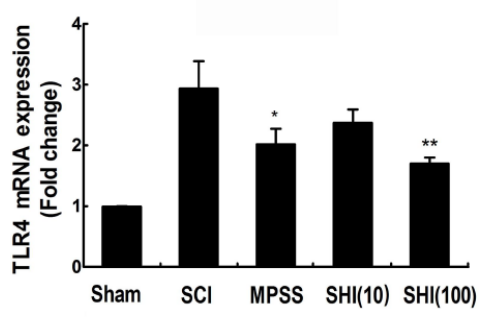

d

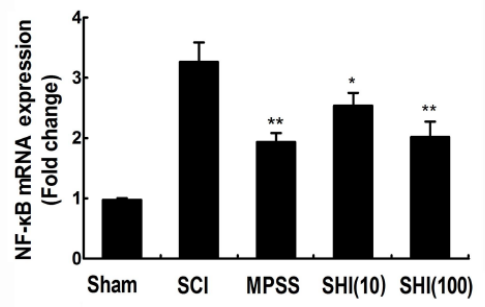

SCI group (Fig. 4c). Moreover, we also found the mRNA expression of NF- $\mathrm{KB}$ in shikonin treatments were significant reduction compared with SCI group (Fig. 4d). Similarly, the protein expression levels of HMGB1, TLR4 and NF- $\kappa$ B in shikonin treatments were obviously decreased compared with SCI group (Fig. 4a). These results revealed shikonin reduced the mRNA and protein expression levels of HMGB1, TLR4 and NF- $\kappa$ B after SCI injury

\section{Shikonin regulated inflammation after SCI injury through HMGB1 signaling}

To investigate the mechanism of shikonin on the repair process after SCI injury, we constructed adenovirus vector containing HMGB1 and compared protein expression levels 
Fig. 5. Shikonin regulated inflammation through HMGB1 signaling. (a) The protein levels of HMGB1, TLR4, $\mathrm{NF}-\kappa \mathrm{B}$ at $72 \mathrm{~h}$ post injury. Quantification data of IL-1 $\beta$ (b), IL-6 (c), TNF- $\alpha$ (d) production in the spinal cord at $72 \mathrm{~h}$ after injury, as assessed by ELISA. Results are expressed as the mean \pm S.D. from three independent experiments. $* \quad \mathrm{P}<0.05$ versus MPSS group, ** $\mathrm{P}<0.01$ versus $\mathrm{SHI}$ group.
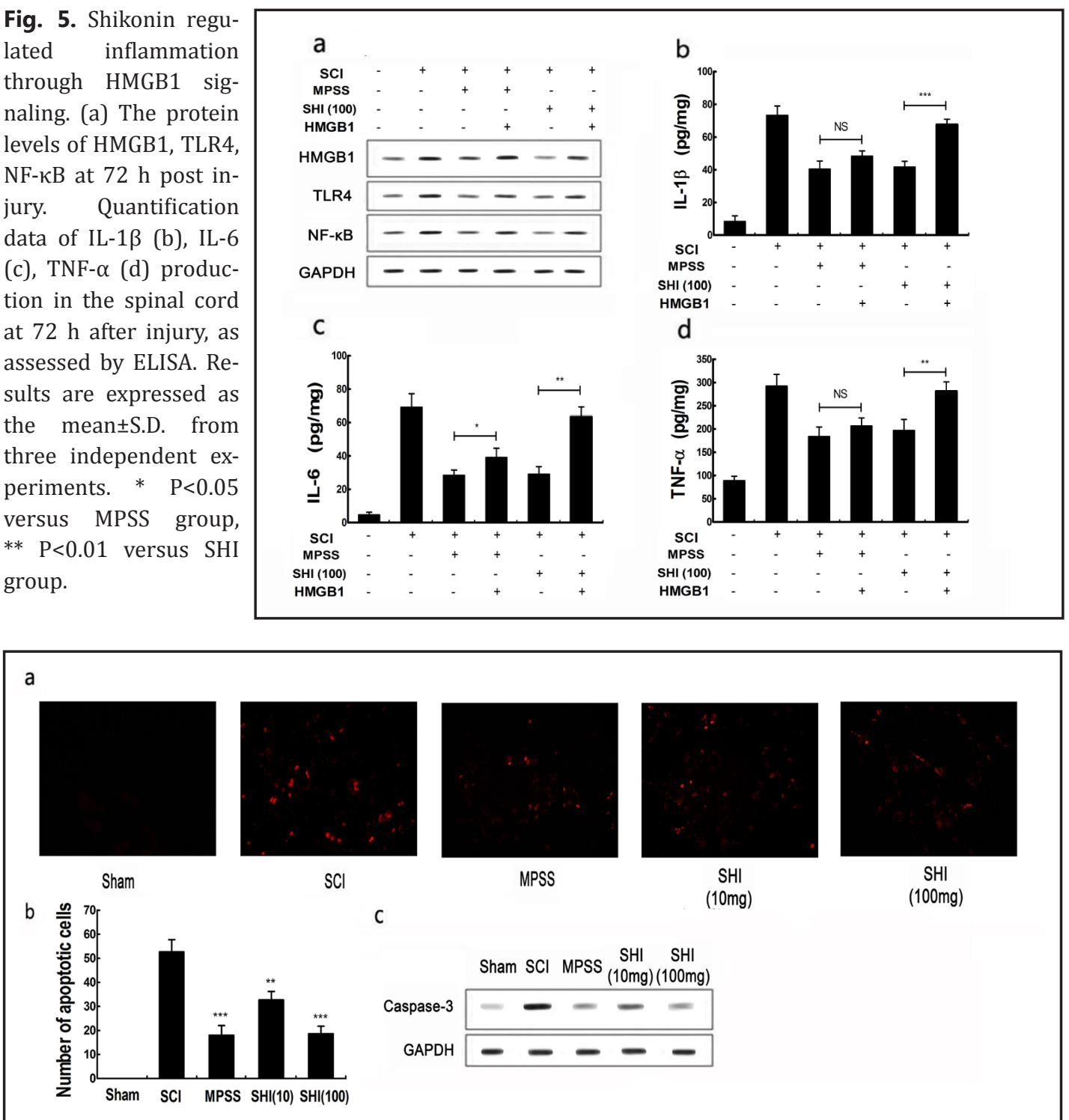

Fig. 6. Effect of shikonin on apoptosis in spinal cord tissue of rats with spinal cordinjury. (a) Apoptotic cells in spinal cord tissue of rats (TUNEL staining, $\times 200$ ), (b) Quantification of apoptotic cells in spinal cord tissue. (c) Shikonin inhibit caspase-3 protein expression. Results are expressed as the mean \pm S.D. from three independent experiments. ${ }^{* *} \mathrm{P}<0.01$ versus $\mathrm{SCI}$ group, *** $\mathrm{P}<0.001$ versus $\mathrm{SCI}$ group.

of HMGB1 signaling pathway in rats after SCI injury. Interestingly, we found that shikonin add overexpression with HMGB1 treatment could obviously increase the HMGB1 signaling protein expression levels including HMGB1, TLR4 and NF-кB compared with shikonin independent treatment (Fig. 5a). Consistently, after overexpression with HMGB1, shikonin treatment group remarkably increased IL-1 $\beta$ (Fig. 5b), IL-6 (Fig. 5c) and TNF- $\alpha$ (Fig. 5d) cytokine levels. However, MPSS add overexpression with HMGB1 treatment could not influence the expression levels of inflammatory cytokines except for IL- 6 secretion $(\mathrm{P}<0.05)$. These results showed shikonin regulated inflammation afer SCI injury in rats through HMGB1 signaling.

Shikonin Inhibits apoptosis of spinal cord in rats after SCI injury

To test the effect of shikonin on the apoptosis of spinal cord in rats, tunel staining was used in each group after SCI injury. We showed the apoptotic cells in SCI group were obviously 


\section{Cellular Physiology and Biochemistry

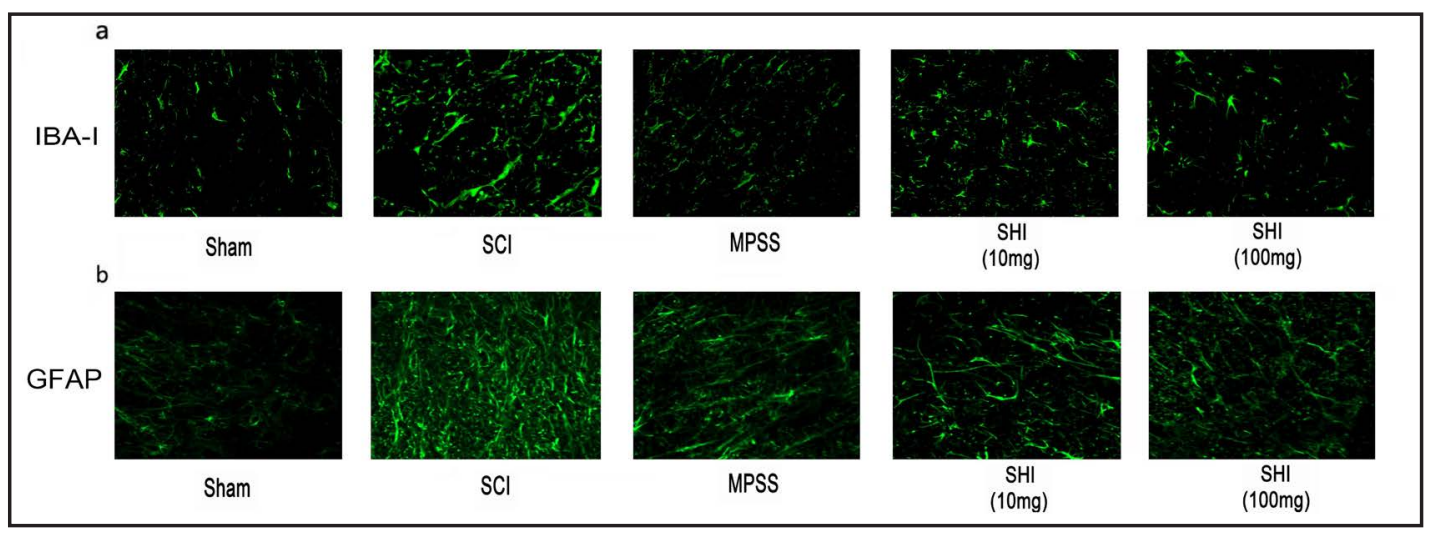

Fig. 7. Effect of shikonin on tissue repair in the spinal cord. (a) Representative photomicrographs of the spinal cord sections of mice subjected to each treatment showing IBA-1 immunoreactive cells at 72 hours after injury. (b) GFAP immunoreactivity to the injury site at 72 hours post-injury.

increased compared with those in sham group (Fig. 6a and 6b). In the group of shikonin treatment following SCI injury, the apoptotic cells in the spinal cord were significantly decreased compared with SCI group. In addition, compared with $10 \mathrm{mg} / \mathrm{kg}$ of shikonin group, $100 \mathrm{mg} / \mathrm{kg}$ of shikonin treatment induced less apoptotic cells after SCI injury. The caspase-3 protein expression level was also detected to identify the degree of apoptosis in spinal cord of rats following SCI injury. Shikonin could decrease the caspase-3 expression in shikonin treatment group compared with SCI group (Fig. 6c). The results suggested that shikonin treatment could inhibit apoptosis in the spinal cord tissue following SCI injury.

\section{Discussion}

The secondary spinal cord injury brings progressive central hemorrhagic necrosis accompanied with inflammatory infiltration [21]. The spinal cord injury induces inflammatory cascades, including TNF- $\alpha$, IL-1 $\beta$ and IL-6 inflammatory cytokines, which promote the acute post-traumatic manner in the spinal cord [22]. The inflammation induced by spinal cord injury extends the actual site of necrosis and apoptosis in the spinal cord, causing more lesions in the extra region and aggravating the dysfunction of spinal cord [23]. The inflammation also further aggravates the tissue degeneration and inhibition of functional recovery in the spinal cord [24]. NF- $\mathrm{BB}$ is one of the most famous inflammatory relevant transcription factors produced by almost all animal cells, which regulates lots of genes and signaling pathways involved in inflammation [25]. NF- $\kappa \mathrm{B}$ is widely studied in the cellular immune response to infection, inflammatory response and autoimmune pathology [26]. NF- $\kappa B$ regulates inflammation through cytokine-directed cell differentiation, including macrophages and lymphocytes [27, 28]. In addition, previous reports revealed proinflammatory cytokines, including TNF- $\alpha$, IL- $1 \beta$ and IL- 6 , could be upregulated by NF- $\kappa$ B $[29,30]$.

This study demonstrated that shikonin could obviously improve the tissue repair and dysfunction of motor at the $100 \mathrm{mg} / \mathrm{kg}$ concentration after SCI in SD rats. Further study revealed shikonin treatment reduced spinal cord edema and inhibited the expression of inflammatory cytokines. Previous study found inflammatory cascades arose from the residential innate immune cells and infiltrating leukocytes in SCI injury [31]. Meanwhile, the profiles of microglia activation and reactive astrocytes were detected distinct from that in the shikonin treatment group. To further explore the protective mechanism of shikonin in SCI rats, the expression levels of HMGB1, TLR4 and NF- $\mathrm{\kappa B}$ were determined and the results showed that the improved tissue repair and decreased inflammation induced by shikonin might 


\section{Cellular Physiology Cell Physiol Biochem 2017;43:481-491

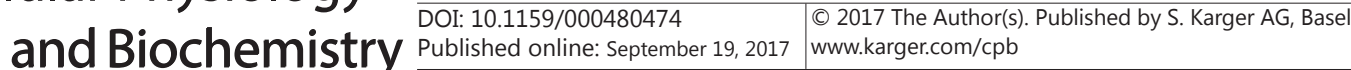 \\ Bi et al.: Molecular Mechanism of Shikonin in Spinal Cord Injury}

be involved in the down-regulation of HMGB1/TLR4/NF- $\mathrm{KB}$ signaling pathway. Previous publication revealed that shikonin could inhibit inflammation and apoptosis through PI3K/ Akt signaling pathway in a osteoarthritis rat model [32]. Latest report indicated that shikonin attenuated cell apoptosis in ConA-induced hepatitis through up-regulating expression of Bcl-2 and reducing expression of Bax, caspase 9 and p-JNK [33]. Consistently, we found that shikonin could improve the recovery of the tissue repair and motor dysfunction after SCI injury. The HE staining showed that shikonin could alleviate tissue injuries, including loose structure, inflammation infiltration and edema after SCI injury.

Previously reported that the mRNA and protein levels of HMGB1 were significantly increased in SCI rats compared with control $[34,35]$. Shikonin treatment could decrease the expression of HMGB1 in RAW264.7 cells [18]. HMGB-1 was mainly expressed by inflammatory cells in SCI injury [17]. Another study also showed that HMGB-1 was expressed in the diabetic rat retinal cells, which might accelerate rat retinal cell inflammation and apoptosis [36]. Here we demonstrated that shikonin administration decreased the production of the HMGB1 mRNA and protein levels after SCI injury in rats. Shindo et al. reported that shikonin could inactivate the NF- $\mathrm{BB}$ pathway in human periodontal ligament cell lines [37]. In addition, previous study reported that HMGB1 promoted the expression levels of NLRP3 and caspase-8 inflammasomes via activation of NF- $\mathrm{KB}$ pathway in acute glaucoma [38]. To further investigate the mechanism effect of HMGB1 decreased by shikonin on the NF- $\kappa B$ signaling pathway in SCI rats, HMGB1 adenovirus vectors were constructed and the results showed that the decreased HMGB1 induced by shikonin down-regulated the NF- $\kappa$ B signaling pathway involving the reduction of TLR4 expression level. Overexpresion of HMGB1 could markedly increase the protein levels of TLR4 and NF- $\kappa \mathrm{B}$ after SCI injury. Moreover, overexpression of HMGB1 could increase the secretion of inflammatory cytokines induced by shikonin in SCI model of rats. Furthermore, we also demonstrated that shikonin administration suppressed the apoptosis of spinal cord tissue after SCI injury model of rats. In addition, we also showed that shikonin could significantly decrease the protein expression level of caspase- 3 after SCI injury model of rats.

Taken together, our results showed that shikonin could induce the recovery of tissue repair and motor function via inactivation of HMGB1/TLR4/ NF- $\kappa B$ signaling pathway in SCI model of rats. Meanwhile, shikonin regulated the inflammation response in SCI was involved in the HMGB1/TLR4/ NF- $\mathrm{\kappa B}$ signaling pathway, and the apoptosis and relevant protein expression inhibited by shikonin were determined to assist the recovery of SCI in rats. This newly mechanism provides further understanding of molecular signaling pathway in spinal cord injury during secondary injury and brings new insight into spinal cord injury. This newly found also could be a novel potential therapeutic manner for the cure of secondary injury in spinal cord injury.

\section{Acknowledgements}

This work was supported by China Postdoctoral Science Foundation (9021346201).

\section{Disclosure Statement}

The authors declare no competing financial interests.

\section{References}

\footnotetext{
1 Wang L, Yao Y, He R, Meng Y, Li N, Zhang D, Xu J, Chen O, Cui J, Bian J, Zhang Y, Chen G, Deng X: Methane ameliorates spinal cord ischemia-reperfusion injury in rats: Antioxidant, anti-inflammatory and antiapoptotic activity mediated by nrf2 activation. Free Radic Biol Med 2017;103:69-86.
} 


\section{Cellular Physiology Cell Physiol Biochem 2017;43:481-491 \begin{tabular}{l|l} 
and Biochemistry & DOI: 10.1159/000480474 \\
(c) 2017 The Author(s). Published by S. Karger AG, Basel \\
www.karger.com/cpb
\end{tabular}

2 Beattie MS: Inflammation and apoptosis: Linked therapeutic targets in spinal cord injury. Trends Mol Med 2004;10:580-583.

-3 Silva NA, Sousa N, Reis RL, Salgado AJ: From basics to clinical: A comprehensive review on spinal cord injury. Prog Neurobiol 2014;114:25-57.

4 Straley KS, Foo CW, Heilshorn SC: Biomaterial design strategies for the treatment of spinal cord injuries. J Neurotrauma 2010;27:1-19.

5 Anwar MA, Al Shehabi TS, Eid AH: Inflammogenesis of secondary spinal cord injury. Front Cell Neurosci 2016;10:98.

6 Zhao H, Cheng L, Du X, Hou Y, Liu Y, Cui Z, Nie L: Transplantation of cerebral dopamine neurotrophic factor transducted bmscs in contusion spinal cord injury of rats: Promotion of nerve regeneration by alleviating neuroinflammation. Mol Neurobiol 2016;53:187-199.

7 Kim Y, Cho HY, Ahn YJ, Kim J, Yoon YW: Effect of nmda nr2b antagonist on neuropathic pain in two spinal cord injury models. Pain 2012;153:1022-1029.

8 Cristante AF, Damasceno ML, Barros Filho TE, de Oliveira RP, Marcon RM, da Rocha ID: Evaluation of the effects of hyperbaric oxygen therapy for spinal cord lesion in correlation with the moment of intervention. Spinal Cord 2012;50:502-506.

9 Deng B, Feng Y, Deng B. TIPE2 mediates the suppressive effects of shikonin on MMP13 in osteosarcoma cells. Cell Physiol Biochem 2015;37(6):2434-2443.

10 Trivedi R, Müller GA, Rathore MS, Mishra DP, Dihazi H. Anti-Leukemic Activity of Shikonin: Role of ERP57 in Shikonin Induced Apoptosis in Acute Myeloid Leukemia. Cell Physiol Biochem 2016;39(2):604-616.

11 Singh B, Sharma MK, Meghwal PR, Sahu PM, Singh S: Anti-inflammatory activity of shikonin derivatives from arnebia hispidissima. Phytomedicine : Phytomedicine 2003;10:375-380.

12 Wang WG, Xiu RJ, Xu ZW, Yin YX, Feng Y, Cao XC, Wang PS: Protective effects of vitamin c against spinal cord injury-induced renal damage through suppression of nf-kappab and proinflammatory cytokines. Neurol Sci 2015;36:521-526.

13 Valaperti A: Drugs targeting the canonical nf-kappab pathway to treat viral and autoimmune myocarditis. Curr Pharm Des 2016;22:440-449.

-14 Andujar I, Recio MC, Bacelli T, Giner RM, Rios JL: Shikonin reduces oedema induced by phorbol ester by interfering with ikappabalpha degradation thus inhibiting translocation of nf-kappab to the nucleus. Br J Pharmacol 2010;160:376-388.

15 Huang CS, Lin AH, Yang TC, Liu KL, Chen HW, Lii CK: Shikonin inhibits oxidized ldl-induced monocyte adhesion by suppressing nfkappab activation via up-regulation of pi3k/akt/nrf2-dependent antioxidation in ea.Hy926 endothelial cells. Biochem Pharmacol 2015;93:352-361.

16 Yang H, Wang H, Czura CJ, Tracey KJ: The cytokine activity of hmgb1. J Leukoc Biol 2005;78:1-8.

17 Kang N, Hai Y, Yang J, Liang F, Gao CJ: Hyperbaric oxygen intervention reduces secondary spinal cord injury in rats via regulation of hmgb1/tlr4/nf-kappab signaling pathway. Int J Clin Exp Pathol 2015;8:1141-1153.

-18 Yang Y, Wang J, Yang Q, Wu S, Yang Z, Zhu H, Zheng M, Liu W, Wu W, He J, Chen Z: Shikonin inhibits the lipopolysaccharide-induced release of hmgb1 in raw264.7 cells via ifn and nf-kappab signaling pathways. Int Immunopharmacol 2014;19:81-87.

19 Hu JZ, Huang JH, Zeng L, Wang G, Cao M, Lu HB: Anti-apoptotic effect of microrna-21 after contusion spinal cord injury in rats. J Neurotrauma 2013;30:1349-1360.

20 Keller AV, Wainwright G, Shum-Siu A, Prince D, Hoeper A, Martin E, Magnuson DS: Disruption of locomotion in response to hindlimb muscle stretch at acute and chronic time points after a spinal cord injury in rats. J Neurotrauma 2017;34:661-670.

21 Fleming JC, Norenberg MD, Ramsay DA, Dekaban GA, Marcillo AE, Saenz AD, Pasquale-Styles M, Dietrich WD, Weaver LC: The cellular inflammatory response in human spinal cords after injury. Brain 2006;129:3249-3269.

22 Geisler FH, Coleman WP, Benzel E, Ducker T, Hurlbert RJ: Spinal cord injury. Lancet 2002;360:1883; Author Reply 1884.

23 Carlson SL, Parrish ME, Springer JE, Doty K, Dossett L: Acute inflammatory response in spinal cord following impact injury. Exp Neurol 1998;151:77-88.

24 Kotipatruni RR, Dasari VR, Veeravalli KK, Dinh DH, Fassett D, Rao JS: P53- and bax-mediated apoptosis in injured rat spinal cord. Neurochem Res 2011;36:2063-2074. 


\section{Cellular Physiology Cell Physiol Biochem 2017;43:481-491

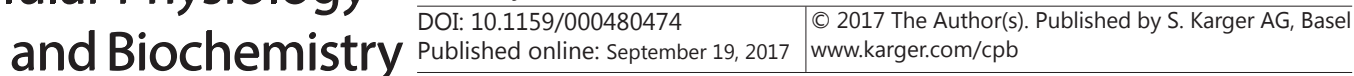 \\ Bi et al.: Molecular Mechanism of Shikonin in Spinal Cord Injury}

-25 Ma B, Hottiger MO: Crosstalk between wnt/beta-catenin and nf-kappab signaling pathway during inflammation. Front Immuno 2016;7:378.

26 Jing H, Lee S: Nf-kappab in cellular senescence and cancer treatment. Mol Cells 2014;37:189-195.

-27 Baker RG, Hayden MS, Ghosh S: Nf-kappab, inflammation, and metabolic disease. Cell Metab 2011;13:1122.

28 Visekruna A, Volkov A, Steinhoff U: A key role for nf-kappab transcription factor c-rel in t-lymphocytedifferentiation and effector functions. Clin Dev Immunol 2012;2012:239368.

29 Wang Y, Sun L, Song Z, Wang D, Bao Y, Li Y: Maspin inhibits macrophage phagocytosis and enhances inflammatory cytokine production via activation of nf-kappab signaling. Mol Immunol 2017;82:94-103.

-30 Zhang H, Zhi L, Moochhala S, Moore PK, Bhatia M: Hydrogen sulfide acts as an inflammatory mediator in cecal ligation and puncture-induced sepsis in mice by upregulating the production of cytokines and chemokines via nf-kappab. Am J Physiol Lung Cell Mol Physiol 2007;292:L960-971.

-31 Anwar MA, Al Shehabi TS, Eid AH: Inflammogenesis of Secondary Spinal Cord Injury. Front Cell Neurosci 2016;13:98.

-32 Fu D, Shang X, Ni Z, Shi G: Shikonin inhibits inflammation and chondrocyte apoptosis by regulation of the pi3k/akt signaling pathway in a rat model of osteoarthritis. Exp Ther Med 2016;12:2735-2740.

33 Liu T, Xia Y, Li J, Li S, Feng J, Wu L, Zhang R, Xu S, Cheng K, Zhou Y, Zhou S, Dai W, Chen K, Wang F, Lu J, Zhou Y, Guo C: Shikonin attenuates concanavalin a-induced acute liver injury in mice via inhibition of the jnk pathway. Mediators Inflamm 2016;2016:2748367.

-34 Yang J, Liu X, Zhou Y, Wang G, Gao C, Su Q: Hyperbaric oxygen alleviates experimental (spinal cord) injury by downregulating hmgb1/nf-kappab expression. Spine 2013;38:E1641-1648.

35 Zhu XL, Chen X, Wang W, Li X, Huo J, Wang Y, Min YY, Su BX, Pei JM: Electroacupuncture pretreatment attenuates spinal cord ischemia-reperfusion injury via inhibition of high-mobility group box 1 production in a lxa4 receptor-dependent manner. Brain Res 2017;1659:113-120.

-36 Yu Y, Yang L, Lv J, Huang X, Yi J, Pei C, Shao Y: The role of high mobility group box 1 (HMGB-1) in the diabetic retinopathy inflammation and apoptosis. Int J Clin Exp Pathol. 2015;8:6807-6813.

37 Shindo S, Hosokawa Y, Hosokawa I, Ozaki K, Matsuo T: Shikonin inhibits inflammatory cytokine production in human periodontal ligament cells. Inflammation 2016;39:1124-1129.

-38 Chi W, Chen H, Li F, Zhu Y, Yin W, Zhuo Y: Hmgb1 promotes the activation of nlrp3 and caspase-8 inflammasomes via nf-kappab pathway in acute glaucoma. J Neuroinflammation 2015;12:137. 